Stud. Univ. Babeş-Bolyai Math. 66(2021), No. 4, 677-690

DOI: 10.24193/subbmath.2021.4.07

\title{
Harmonic mappings and its directional convexity
}

\author{
Poonam Sharma and Omendra Mishra
}

\begin{abstract}
For any $\mu_{j}\left(\mu_{j} \in \mathbb{C},\left|\mu_{j}\right|=1, j=1,2\right)$, we consider the rotations $f_{\mu_{1}}$ and $F_{\mu_{2}}$ of right half-plane harmonic mappings $f, F \in S_{\mathcal{H}}$ which are CHD with the prescribed dilatations $\omega_{f}(z)=(a-z) /(1-a z)$ for some $a(-1<a<1)$ and $\omega_{F}(z)=e^{i \theta} z^{n}(n \in \mathbb{N}, \theta \in \mathbb{R}), \omega_{F}(z)=(b-z) /(1-b z)$, $\omega_{F}(z)=\left(b-z e^{i \phi}\right) /\left(1-b z e^{i \phi}\right)(-1<b<1, \phi \in \mathbb{R})$, respectively. It is proved that the convolution $f_{\mu_{1}} * F_{\mu_{2}} \in S_{\mathcal{H}}$ and is convex in the direction of $\overline{\mu_{1} \mu_{2}}$ under certain conditions on the parameters involved.
\end{abstract}

Mathematics Subject Classification (2010): 31A05, 30C45, 30C55.

Keywords: Harmonic functions, half-plane mappings, convexity in one direction, harmonic convolution, directional convexity, Rouche's theorem.

\section{Introduction and preliminaries}

Let $\mathcal{H}$ denotes the class of complex-valued functions $f=u+i v$ which are harmonic in the unit disk $\mathbb{D}=\{z \in \mathbb{C}:|z|<1\}$, where $u$ and $v$ are real-valued harmonic functions in $\mathbb{D}$. A function $f \in \mathcal{H}$ can also be expressed as $f=h+\bar{g}$, where $h$ and $g$ are analytic in $\mathbb{D}$, and are called the analytic and co-analytic parts of $f$, respectively. The Jacobian of the function $f=h+\bar{g}$ is given by $J_{f}(z)=\left|h^{\prime}(z)\right|^{2}-\left|g^{\prime}(z)\right|^{2}$. According to the Lewy's [8], every harmonic function $f=h+\bar{g} \in \mathcal{H}$ is locally univalent and sense preserving in $\mathbb{D}$ if and only if $J_{f}(z)>0$ in $\mathbb{D}$ which is equivalent to the existence of an analytic function $\omega_{f}(z)=g^{\prime}(z) / h^{\prime}(z)$ in $\mathbb{D}$ such that $\left|\omega_{f}(z)\right|<1$ for all $z \in \mathbb{D}$. The function $\omega_{f}(z)$ is called the dilatation of the function $f$. The class of all univalent, sense preserving harmonic functions $f=h+\bar{g} \in \mathcal{H}$, normalized by the conditions $h(0)=0=g(0)$ and $h^{\prime}(0)=1$ is denoted by $S_{\mathcal{H}}$. If the function $f=h+\bar{g} \in S_{\mathcal{H}}$, then the functions $h$ and $g$ are of the form:

$$
h(z)=z+\sum_{n=2}^{\infty} a_{n} z^{n} \quad \text { and } \quad g(z)=\sum_{n=1}^{\infty} b_{n} z^{n} \quad\left(\left|b_{1}\right|<1\right) .
$$

The subclass of functions $f=h+\bar{g} \in S_{\mathcal{H}}$ satisfying condition $g^{\prime}(0)=0$ (or equivalently $\left.\omega_{f}(0)=0\right)$ is denoted by $S_{\mathcal{H}}^{0}$. Further, the subclasses of convex, close to convex 
functions $f$ in $S_{\mathcal{H}}\left(\right.$ or $\left.S_{\mathcal{H}}^{0}\right)$ are denoted, respectively, by $K_{\mathcal{H}}, C_{\mathcal{H}}\left(\right.$ or $\left.K_{\mathcal{H}}^{0}, C_{\mathcal{H}}^{0}\right)$. The convolution of two analytic functions

$$
f(z)=\sum_{n=1}^{\infty} a_{n} z^{n} \text { and } g(z)=\sum_{n=1}^{\infty} b_{n} z^{n}
$$

is defined by

$$
(f * g)(z)=\sum_{n=1}^{\infty} a_{n} b_{n} z^{n} .
$$

The convolution of two harmonic functions $f=h+\bar{g}$ and $F=H+\bar{G}$ is defined by

$$
(f * F)(z)=g * G+\overline{(h * H)} .
$$

A domain $\Omega \subset \mathbb{C}$ is said to be convex in the direction $e^{i \gamma}(\gamma \in \mathbb{R})$, if for every $t \in \mathbb{C}$, the set $\Omega \cap\left\{t+r e^{i \gamma}: r \in \mathbb{R}\right\}$ is either connected or empty. In particular, a domain is convex in the horizontal direction CHD if every line parallel to the real axis has a connected or empty intersection with $\Omega$. Clunie and Sheil-Small [2] introduced shear construction method which provides a univalent harmonic function from a related analytic function and this fundamental theorem is the following:

Theorem 1.1. [2]A locally univalent harmonic function $f=h+\bar{g}$ is a univalent mapping of $\mathbb{D}$ onto a domain convex in the direction of $e^{i \gamma}$ if and only if $h-e^{2 i \gamma} g$ is a analytic univalent mapping of $\mathbb{D}$ onto a domain convex in the direction of $e^{i \gamma}$.

We may construct a harmonic function $f=h+\bar{g} \in S_{\mathcal{H}}$, where $h$ and $g$ are of the form (1.1) by the shearing of a normalized analytic function $(h-g) /\left(1-b_{1}\right)$ which is univalent. Throughout the paper we take $b_{1}=-a(-1<a<1)$.

Definition 1.2 (Slanted and right half-planes). The region $H_{\mu}^{a}$ for some $\mu(\mu \in \mathbb{C}$, $|\mu|=1)$ and for some $a(-1<a<1)$ defined by

$$
H_{\mu}^{a}:=\left\{w \in \mathbb{C}: \Re(\mu w)>-\frac{1+a}{2}\right\}
$$

is called a slanted half-plane and the region $H_{1}^{a}=: H^{a}(-1<a<1)$ is the right half-plane. When $\mu=e^{i \gamma}$ for $\gamma \in[0,2 \pi)$, we denote the region $H_{\mu}^{a}$ by $\mathcal{H}_{\gamma}^{a}$.

The class $S\left(H_{\mu}^{a}\right)$ consists of functions $f \in S_{\mathcal{H}}$ which map $\mathbb{D}$ onto a slanted halfplane $H_{\mu}^{a}$ and in particular, $S\left(H^{a}\right)$ denotes a class of functions $f \in S_{\mathcal{H}}$ which are the right half-plane mappings. If $\mu=e^{i \gamma}$ for $\gamma \in[0,2 \pi)$, then the class $S\left(H_{\mu}^{a}\right)$ is denoted by $S\left(\mathcal{H}_{\gamma}^{a}\right)$. Also, if $a=0$, then the class $S\left(\mathcal{H}_{\gamma}^{a}\right)$ is denoted by $S^{0}\left(\mathcal{H}_{\gamma}\right)$ (see Dorff et al. [4].)

Definition 1.3 (Rotation by $\mu$ ). The rotations of the function $f$ by $\mu(\mu \in \mathbb{C},|\mu|=1)$, denoted by $f_{\mu}$ is given by

$$
f_{\mu}(z)=\bar{\mu} f(\mu z)
$$

The convolution of two analytic convex mappings is convex. However the convolution of two convex harmonic functions need not be convex under convolution. Therefore, it is interesting to study convolution properties of harmonic functions. 
Convolution of harmonic functions convex in the given direction has also been studied in $[1,3,4,5,6,7,9,10,12,13]$. In this work rotations of right half-plane harmonic mappings which are CHD are defined and the convolution of these rotations are studied unlike the earlier studies $[4,10,11,13]$, where the convolution of slanted half-plane harmonic mappings which are convex in certain directions are studied. Indeed, we study the convolution $f_{\mu_{1}} * F_{\mu_{2}}$, where for complex number $\mu_{j}$ with $\left|\mu_{j}\right|=1, j=1,2, f_{\mu_{1}}$ and $F_{\mu_{2}}$ are the rotations of right half-plane harmonic mappings $f, F \in S_{\mathcal{H}}$ which are CHD with dilations $\omega_{f}(z)=(a-z) /(1-a z),(-1<a<1)$, $\omega_{F}(z)=e^{i \theta} z^{n},(n \in \mathbb{N}, \theta \in \mathbb{R}), \omega_{F}(z)=(b-z) /(1-b z),(-1<b<1)$ and $\omega_{F}(z)=\left(b-z e^{i \phi}\right) /\left(1-b z e^{i \phi}\right),(-1<b<1, \phi \in \mathbb{R})$, respectively. It is proved that the convolution $f_{\mu_{1}} * F_{\mu_{2}} \in S_{\mathcal{H}}$ and is convex in the direction of $\overline{\mu_{1} \mu_{2}}$ under certain conditions on the parameters involved.

\section{Preliminaries}

We need following lemmas in proving our results.

Lemma 2.1. [14] Let the function $f: \mathbb{D} \rightarrow \mathbb{C}$ be an analytic function with $f(0)=0$ and $f^{\prime}(0) \neq 0$. Suppose that

$$
\varphi(z)=\frac{z}{\left(1+z e^{i \theta}\right)\left(1+z e^{-i \theta}\right)} \quad(\theta \in \mathbb{R} ; z \in \mathbb{D}) .
$$

If the function $f$ satisfy

$$
\Re\left(\frac{z f^{\prime}(z)}{\varphi(z)}\right)>0 \quad(z \in \mathbb{D}),
$$

then the function $f$ is convex in the direction of real axis.

Lemma 2.2. (Cohn's rule [15, p. 375]) For a polynomial p given by

$$
p(z)=p_{0}(z)=a_{n} z^{n}+a_{n-1} z^{n-1}+\cdots+a_{1} z+a_{0} \quad\left(a_{n} \neq 0\right)
$$

of degree $n$, let $p^{*}$ be an associated polynomial given by

$$
p^{*}(z)=p_{0}^{*}(z)=z^{n} \overline{p\left(\frac{1}{\bar{z}}\right)}=\overline{a_{n}}+\overline{a_{n-1}} z+\cdots+\overline{a_{1}} z^{n-1}+\overline{a_{0}} z^{n} .
$$

Denote by $r$ and $s$ the number of zeroes of the polynomial inside the unit circle and on it, respectively. If $\left|a_{0}\right|<\left|a_{n}\right|$, then the polynomial $p_{1}$ is given by

$$
p_{1}(z)=\frac{\overline{a_{n}} p(z)-a_{0} p^{*}(z)}{z}
$$

is of degree $n-1$ with $r_{1}=r-1$ and $s_{1}=s$ the number of zeroes of $p_{1}$ inside the unit circle and on it, respectively.

We first mention the following result which can be proved by using the definition of rotation and [2, Theorem 1.1].

Lemma 2.3. If the function $f=h+\bar{g} \in S_{\mathcal{H}}$ is CHD, then for any $\mu(\mu \in \mathbb{C},|\mu|=1)$, $f_{\mu}=H+\bar{G} \in S_{\mathcal{H}}$ is convex in the direction of $\bar{\mu}$, where

$$
H(z)=\bar{\mu} h(\mu z) \text { and } G(z)=\mu g(\mu z) .
$$


Lemma 2.4. Let the function $f=h+\bar{g} \in S\left(H^{a}\right)$. Then for any $\mu(\mu \in \mathbb{C},|\mu|=1)$, $f_{\mu}=H+\bar{G} \in S\left(H_{\mu}^{a}\right)$, where $H$ and $G$ are given by (2.3) and hence

$$
H(z)+\bar{\mu}^{2} G(z)=\frac{(1+a) z}{1-\mu z} \quad(z \in \mathbb{D}) .
$$

In particular,

$$
h(z)+g(z)=\frac{(1+a) z}{1-z} \quad(z \in \mathbb{D}) .
$$

The proof of Lemma 2.4 is similar to the proof of [4, Theorem 1.1]

Lemma 2.5. Let for each $j=1$, 2, let the function $f_{j}=h_{j}+\overline{g_{j}} \in S\left(H^{a_{j}}\right)$ be CHD maps and the function $f=f_{1} * f_{2} \in S_{\mathcal{H}}$. Then for any $\mu_{j}\left(\mu_{j} \in \mathbb{C},\left|\mu_{j}\right|=1, j=1,2\right)$, $f_{\mu_{j}} \in S\left(H_{\mu_{j}}^{a_{j}}\right)$ is convex in the direction of $\overline{\mu_{j}}$ and $f_{\mu_{1}} * f_{\mu_{2}}=f_{\mu_{1} \mu_{2}} \in S_{\mathcal{H}}$ is convex in the direction of $\overline{\mu_{1} \mu_{2}}$ in $\mathbb{D}$.

Proof. Let, for each $j=1,2$, the function $f_{j}=h_{j}+\overline{g_{j}} \in S\left(H^{a_{j}}\right)$ be CHD maps. Then, by Theorem $1.1, F_{j}:=h_{j}-g_{j}$ is CHD and, from (2.5) of Lemma 2.4

$$
h_{j}(z)+g_{j}(z)=\frac{\left(1+a_{j}\right) z}{1-z} \quad(z \in \mathbb{D}) .
$$

Then for any $\mu_{j}\left(\mu_{j} \in \mathbb{C},\left|\mu_{j}\right|=1, j=1,2\right), f_{\mu_{j}}=H_{j}+\overline{G_{j}} \in S\left(H_{\mu_{j}}^{a_{j}}\right)$ and is convex in the direction of $\overline{\mu_{j}}$ by Lemma 2.3 , where, in view of (2.3),

$$
H_{j}(z)=\overline{\mu_{j}} h_{j}\left(\mu_{j} z\right) \text { and } G_{j}(z)=\mu_{j} g_{j}\left(\mu_{j} z\right) \text {. }
$$

Since the function $f=f_{1} * f_{2}=h+\bar{g}$ we have

$$
\begin{aligned}
f_{\mu_{1}} * f_{\mu_{2}} & =H_{1} * H_{2}+\overline{G_{1} * G_{2}} \\
& =\overline{\mu_{1} \mu_{2}}\left(h_{1} * h_{2}\right)\left(\mu_{1} \mu_{2} z\right)+\overline{\mu_{1} \mu_{2}\left(g_{1} * g_{2}\right)\left(\mu_{1} \mu_{2} z\right)} \\
& =\overline{\mu_{1} \mu_{2}} h\left(\mu_{1} \mu_{2} z\right)+\overline{\mu_{1} \mu_{2} g\left(\mu_{1} \mu_{2} z\right)} \\
& =f_{\mu_{1} \mu_{2}}:=H+\bar{G} .
\end{aligned}
$$

Since $f=f_{1} * f_{2} \in S_{\mathcal{H}}$, by Lemma $2.3, f_{\mu_{1}} * f_{\mu_{2}}=f_{\mu_{1} \mu_{2}} \in S_{\mathcal{H}}$. We now show that $f_{\mu_{1} \mu_{2}}$ is convex in the direction of $\overline{\mu_{1} \mu_{2}}$. In view of Lemma 2.3, it is enough to prove that $f=h+\bar{g}$ is CHD or by Theorem 1.1, $h-g$ is CHD. Since $h=h_{1} * h_{2}$ and $g=g_{1} * g_{2}$, we have

$$
\begin{aligned}
F_{1} & =\left(h_{1}-g_{1}\right) *\left(h_{2}+g_{2}\right) \\
& =h_{1} * h_{2}+h_{1} * g_{2}-g_{1} * h_{2}-g_{1} * g_{2}
\end{aligned}
$$

and

$$
\begin{aligned}
F_{2} & =\left(h_{2}-g_{2}\right) *\left(h_{1}+g_{1}\right) \\
& =h_{1} * h_{2}+g_{1} * h_{2}-h_{1} * g_{2}-g_{1} * g_{2},
\end{aligned}
$$

where from (2.6),

$$
F_{1}=\left(h_{1}(z)-g_{1}(z)\right) * \frac{\left(1+a_{2}\right) z}{1-z}=\left(1+a_{2}\right)\left(h_{1}(z)-g_{1}(z)\right)
$$


and

$$
F_{2}=\left(h_{2}(z)-g_{2}(z)\right) * \frac{\left(1+a_{1}\right) z}{1-z}=\left(1+a_{1}\right)\left(h_{2}(z)-g_{2}(z)\right) .
$$

Then from (2.7) and (2.8),

$$
\frac{1}{2}\left(F_{1}+F_{2}\right)=h_{1} * h_{2}-g_{1} * g_{2} .
$$

Hence, we only need to prove that $F_{1}+F_{2}$ is CHD. We have from (2.9) and (2.10)

$$
F_{1}+F_{2}=\left(1+a_{2}\right)\left(h_{1}(z)-g_{1}(z)\right)+\left(1+a_{1}\right)\left(h_{2}(z)-g_{2}(z)\right)
$$

and

$$
z\left(F_{1}+F_{2}\right)^{\prime}(z)=\left(1+a_{2}\right) z\left(h_{1}^{\prime}(z)+g_{1}^{\prime}(z)\right) p_{1}(z)+\left(1+a_{1}\right) z\left(h_{2}^{\prime}(z)+g_{2}^{\prime}(z)\right) p_{2}(z)
$$

where for each $j=1,2$,

$$
p_{i}(z)=\frac{h_{i}^{\prime}(z)-g_{i}^{\prime}(z)}{h_{i}^{\prime}(z)+g_{i}^{\prime}(z)}
$$

Since $f_{i} \in S_{H}$, the dilatation $\omega_{f_{i}}=g_{i} / f_{i}$ satisfy $\left|\omega_{f_{i}}\right|<1$ and hence

$$
\Re\left(p_{i}(z)\right)=\Re\left(\frac{1-\omega_{f_{i}}(z)}{1+\omega_{f_{i}}(z)}\right)>0 .
$$

Hence, on using the derivative of (2.6), in (2.11) we have

$$
z\left(F_{1}+F_{2}\right)^{\prime}(z)=\left(1+a_{2}\right)\left(1+a_{1}\right) \frac{z}{(1-z)^{2}}\left[p_{1}(z)+p_{2}(z)\right]
$$

and with $\varphi(z)=\frac{z}{(1-z)^{2}}$, we have

$$
\Re\left(\frac{z\left(F_{1}+F_{2}\right)^{\prime}(z)}{\varphi(z)}\right)=\left(1+a_{2}\right)\left(1+a_{1}\right) \Re\left(p_{1}(z)+p_{2}(z)\right)>0 \quad(z \in \mathbb{D}) .
$$

By Lemma 2.1 it follows that $F_{1}+F_{2}$ is CHD and hence, its harmonic shear $f$ is CHD and by Lemma $2.3, f_{\mu_{1} \mu_{2}}$ is convex in the direction of $\overline{\mu_{1} \mu_{2}}$ in $\mathbb{D}$. This completes the proof.

A equivalent form of Lemma 2.5 is as follows:

Lemma 2.6. Let for each $j=1,2, f_{j}=h_{j}+\overline{g_{j}} \in S\left(H^{a_{j}}\right)$ be CHD and let $f_{\gamma_{j}}$ be the rotations of $f_{j}$ by $e^{i \gamma_{j}}$. Then $f_{\gamma_{j}} \in S\left(\mathcal{H}_{\gamma_{j}}^{a_{j}}\right)$ is convex in the direction of $e^{-i \gamma_{j}}$ and $f_{\gamma_{1}} * f_{\gamma_{2}} \in S_{\mathcal{H}}$ and is convex in the direction of $-\left(\gamma_{1}+\gamma_{2}\right)$ if $f_{1} * f_{2} \in S_{\mathcal{H}}$.

Our next lemma gives a formula for the dilatation of the convolution if two slanted right-half plane mapping.

Lemma 2.7. Let the function $f=h+\bar{g} \in S\left(H^{a}\right)$ with the dilatation

$$
\omega_{f}(z)=(a-z) /(1-a z)(-1<a<1)
$$

and let the function $F=H+\bar{G} \in S\left(H^{b}\right)$ with a dilatation $\omega_{F}$. Then the dilatation $\tilde{\omega}(z)$ of the convolution $f * F$ is given by

$$
\tilde{\omega}(z)=\frac{2(a-z) \omega_{F}(z)\left(1+\omega_{F}(z)\right)-(1-a) z(1-z) \omega_{F}^{\prime}(z)}{2(1-a z)\left(1+\omega_{F}(z)\right)-(1-a) z(1-z) \omega_{F}^{\prime}(z)} .
$$


Proof. Since $f=h+\bar{g} \in S\left(H^{a}\right)$ and the dilatation $\omega_{f}(z)=(a-z) /(1-a z)$, we obtain from (2.5) that

$$
h^{\prime}(z)=\frac{1-a z}{(1-z)^{3}} \quad \text { and } \quad g^{\prime}(z)=\frac{a-z}{(1-z)^{3}} .
$$

which gives

$$
h(z)=\frac{1+a}{2}\left[\frac{z}{1-z}+\frac{(1-a) z}{(1+a)(1-z)^{2}}\right]
$$

and

$$
g(z)=\frac{1+a}{2}\left[\frac{z}{1-z}-\frac{(1-a) z}{(1+a)(1-z)^{2}}\right] .
$$

Using (2.13) and (2.14) the dilatation $\tilde{\omega}(z)$ of $f * F$ is given by

$$
\tilde{\omega}(z)=\frac{(g * G)^{\prime}(z)}{(h * H)^{\prime}(z)}=\frac{2 a G^{\prime}(z)-(1-a) z G^{\prime \prime}(z)}{2 H^{\prime}(z)+(1-a) z H^{\prime \prime}(z)} .
$$

Since $G^{\prime}(z)=\omega_{F}(z) H^{\prime}(z)$, we have $G^{\prime \prime}(z)=\omega_{F}^{\prime}(z) H^{\prime}(z)+\omega_{F}(z) H^{\prime \prime}(z)$ and, from $(2.5)$

$$
H(z)+G(z)=\frac{(1+b) z}{1-z} \quad(z \in \mathbb{D})
$$

which in turn gives

$$
H^{\prime}(z)=\frac{1+b}{\left(1+\omega_{F}(z)\right)(1-z)^{2}}
$$

and

$$
H^{\prime \prime}(z)=\frac{(1+b)\left[2\left(1+\omega_{F}(z)\right)-(1-z) \omega_{F}^{\prime}(z)\right]}{\left(1+\omega_{F}(z)\right)^{2}(1-z)^{3}} .
$$

Hence, from (2.15), we obtain

$$
\tilde{\omega}(z)=\frac{\left\{2 a \omega_{F}(z)-(1-a) z \omega_{F}^{\prime}(z)\right\} H^{\prime}(z)-(1-a) z \omega_{F}(z) H^{\prime \prime}(z)}{2 H^{\prime}(z)+(1-a) z H^{\prime \prime}(z)}
$$

On using (2.17) and (2.18), the desired expression for $\tilde{\omega}(z)$ follows.

A particular form of Lemma 2.7 is as follows:

Corollary 2.8. Let the function $f=h+\bar{g} \in S\left(H^{0}\right)$ with the dilatation $\omega_{f}(z)=-z$ and let the function $F=H+\bar{G} \in S\left(H^{b}\right)$ with a dilatation $\omega_{F}(z)$. Then the dilatation $\tilde{\omega}(z)$ of the convolution $f * F$ is given by

$$
\tilde{\omega}(z)=\frac{-2 z \omega_{F}(z)\left(1+\omega_{F}(z)\right)-z(1-z) \omega_{F}^{\prime}(z)}{2\left(1+\omega_{F}(z)\right)-z(1-z) \omega_{F}^{\prime}(z)} .
$$




\section{Main results}

We now prove our first main result on convolution $f_{\mu_{1}} * F_{\mu_{2}}$, where the function $f=h+\bar{g} \in S\left(H^{a}\right)$ with the dilatation $\omega_{f}(z)=(a-z) /(1-a z)(-1<a<1)$ and $F=H+\bar{G} \in S\left(H^{0}\right)$ with the dilatation $\omega_{F}=e^{i \theta} z^{n}(n \in \mathbb{N}, \theta \in \mathbb{R})$ which is as below:

Theorem 3.1. Let the function $f=h+\bar{g} \in S\left(H^{a}\right)$ and the function

$$
F=H+\bar{G} \in S\left(H^{0}\right)
$$

be CHD maps with the dilatations

$$
\omega_{f}(z)=(a-z) /(1-a z) \quad(-1<a<1) \text { and } \omega_{F}(z)=e^{i \theta} z^{n} \quad(n \in \mathbb{N}, \theta \in \mathbb{R}),
$$

respectively, then for any $\mu_{j}\left(\mu_{j} \in \mathbb{C},\left|\mu_{j}\right|=1, j=1,2\right)$, the function $f_{\mu_{1}} * F_{\mu_{2}} \in S_{\mathcal{H}}$ is convex in the direction of $\overline{\mu_{1} \mu_{2}}$ for $a \in[(n-2) /(n+2), 1)$.

Proof. In view of Lemma 2.5, it is enough to show that $f * F \in S_{\mathcal{H}}$. Let $\tilde{\omega}(z)$ be the dilatation of the convolution $f * F$. Since $\omega_{F}(z)=e^{i \theta} z^{n}(n \in \mathbb{N}, \theta \in \mathbb{R})$ in (2.12) of Lemma 2.7, gives

$$
\tilde{\omega}(z)=\frac{2(a-z) e^{i \theta} z^{n}\left(1+e^{i \theta} z^{n}\right)-(1-a) z(1-z) n e^{i \theta} z^{n-1}}{2(1-a z)\left(1+e^{i \theta} z^{n}\right)-(1-a) z(1-z) n e^{i \theta} z^{n-1}}=-z^{n} e^{2 i \theta} \frac{p(z)}{p^{*}(z)},
$$

where

$$
p(z)=p_{0}(z)=z^{n+1}-a z^{n}+\frac{1}{2}(2+a n-n) e^{-i \theta} z+\frac{1}{2}(n-2 a-a n) e^{-i \theta}
$$

and

$$
p^{*}(z)=z^{n+1} \overline{p\left(\frac{1}{\bar{z}}\right)}
$$

Let $A_{1}, A_{2}, A_{3}, \cdots, A_{n+1}$ be the zeros ( not necessarily distinct) of the polynomial $p$, so that $1 / \overline{A_{1}}, 1 / \overline{A_{2}}, 1 / \overline{A_{3}}, \ldots, 1 \overline{A_{n+1}}$ are the zeros of the polynomial $p^{*}$, Then, it follows that

$$
\tilde{\omega}(z)=-z^{n} e^{2 i \theta} \frac{\left(z-A_{1}\right)\left(z-A_{2}\right) \cdots\left(z-A_{n+1}\right)}{\left(1-\overline{A_{1}} z\right)\left(1-\overline{A_{2}} z\right) \cdots\left(1-\overline{A_{n+1}} z\right)} .
$$

Now, we only need to prove that $|\tilde{\omega}(z)|<1$. If $a=(n-2) /(n+2)$, then

$$
p(z)=e^{-i \theta}\left[z^{n+1} e^{i \theta}-\frac{n-2}{n+2} z^{n} e^{i \theta}-\frac{n-2}{n+2} z+1\right]
$$

and

which proves that

$$
p^{*}(z)=z^{n+1} e^{i \theta}-\frac{n-2}{n+2} z^{n} e^{i \theta}-\frac{n-2}{n+2} z+1
$$

$$
|\tilde{\omega}(z)|=\left|-z^{n} e^{i \theta}\right|<1 .
$$

Let $(n-2) /(n+2)<a<1$. We first show that each zero $A_{i}$ of $p$ lies inside and on the unit circle: $\left|A_{i}\right| \leq 1$ for each $i=1,2, \ldots, n+1$. We apply the Cohn's rule to the polynomial $p$ of degree $n+1$ given by (3.1). Since

$$
\left|a_{0}\right|:=\left|\frac{1}{2}(n-2 a-a n) e^{-i \theta}\right|<1=:\left|a_{n+1}\right|
$$


and

we see that

$$
\frac{(1-a)(2+n)[2(1+a)-(1-a) n]}{4}>0,
$$

$$
\begin{aligned}
p_{1}(z) & =\frac{\overline{a_{n+1}} p(z)-a_{0} p^{*}(z)}{z} \\
& =\frac{(1-a)(2+n)[2(1+a)-(1-a) n]}{4} q_{1}(z),
\end{aligned}
$$

and hence, $p_{1}(z)$ has same same zeros as the polynomial

$$
q_{1}(z)=z^{n}-\frac{n}{n+2} z^{n-1}+\frac{2}{n+2} e^{-i \theta}
$$

has. If $n=1, q_{1}(z)$ has a zero at

$$
z=\frac{1}{3}-\frac{2}{3} e^{-i \theta}
$$

which lies inside or on the unit circle $|z|=1$. Hence, $p_{1}(z)$ has a zero inside or on the unit circle $|z|=1$ if $-\frac{1}{3}<a<1$. If $n \geq 2$ and if we write Eq. (3.3) as

$$
q_{1}(z)=q_{2}(z)+q_{3}(z) \text {, }
$$

where $q_{2}(z)=z^{n}$ and

$$
q_{3}(z)=-\frac{n}{n+2} z^{n-1}+\frac{2}{n+2} e^{-i \theta},
$$

then on $|z|=1+\epsilon(\epsilon>0)$,

$$
\left|q_{2}(z)\right|=(1+\epsilon)^{n}
$$

and

Therefore, we have

$$
\left|q_{3}(z)\right| \leq \frac{n}{n+2}(1+\epsilon)^{n-1}+\frac{2}{n+2}
$$

on $|z|=1+\epsilon$ if

$$
\left|q_{3}(z)\right|<\left|q_{2}(z)\right|
$$

$$
\frac{n}{n+2}(1+\epsilon)^{n-1}+\frac{2}{n+2}<(1+\epsilon)^{n}
$$

Since $(1+\epsilon)^{n-1}>1$,

$$
\frac{n}{n+2}(1+\epsilon)^{n-1}+\frac{2}{n+2}<\frac{n+2}{n+2}(1+\epsilon)^{n-1}=(1+\epsilon)^{n-1}<(1+\epsilon)^{n},
$$

we have

$$
\left|q_{3}(z)\right|<\left|q_{2}(z)\right| \text { on }|z|=1+\epsilon
$$

and hence, by well known Rouche's Theorem the polynomials $q_{2}$ and $q_{2}+q_{3}=q_{1}$ have same number of zeros inside the disk $|z|<1+\epsilon$. As the polynomial $q_{2}$ has $n$ zeros inside the disk $|z|<1+\epsilon$, the polynomial $q_{1}$ has $n$ zeroes in that disk. Letting $\epsilon \rightarrow 0$, we obtain that the polynomial $q_{1}$ has all of its $n$ zeros in the disk $|z| \leq 1$. It proves consequently that all the zeros $A_{i}$ of $p(z)$ lie inside or on the unit circle $|z|=1$. This proves the result. 
Our second main result on convolution $f_{\mu_{1}} * F_{\mu_{2}}$ of rotation of $f=h+\bar{g} \in S\left(H^{a}\right)$ with the dilatation $\omega_{f}(z)=(a-z) /(1-a z)(-1<a<1)$ and the function $F=H+\bar{G} \in$ $S\left(H^{b}\right)$ with the dilatation $\omega_{F}(z)=(b-z) /(1-b z)(-1<b<1)$ is as below:

Theorem 3.2. Let the function $f=h+\bar{g} \in S\left(H^{a}\right)$ and the function

$$
F=H+\bar{G} \in S\left(H^{b}\right)
$$

be CHD maps with the dilatation

$$
\omega_{f}(z)=(a-z) /(1-a z) \quad(-1<a<1)
$$

and

$$
\omega_{F}(z)=(b-z) /(1-b z)(-1<b<1)
$$

respectively, then for any $\mu_{j}\left(\mu_{j} \in \mathbb{C},\left|\mu_{j}\right|=1, j=1,2\right), f_{\mu_{1}} * F_{\mu_{2}} \in S_{\mathcal{H}}$ is convex in the direction of $\overline{\mu_{1} \mu_{2}}$ provided $(a+b) /(1+a b) \in[-1 / 3,1)$.

Proof. To prove the result, in view of Lemma 2.5, we prove that $f * F \in S_{\mathcal{H}}$. Let $\tilde{\omega}(z)$ be the dilatation of $f * F$ when $\omega_{F}(z)=(b-z) /(1-b z)(-1<b<1)$. Then from Lemma 2.7 we have

$$
\tilde{\omega}(z)=\frac{2(a-z) \frac{b-z}{1-b z}\left(1+\frac{b-z}{1-b z}\right)-(1-a) z(1-z) \frac{b^{2}-1}{(1-b z)^{2}}}{2(1-a z)\left(1+\frac{b-z}{1-b z}\right)-(1-a) z(1-z) \frac{b^{2}-1}{(1-b z)^{2}}}=\frac{r(z)}{r^{*}(z)},
$$

where

$$
r(z)=z^{2}+\frac{1}{2}(a b-3 a-3 b+1) z+a b
$$

and

$$
r^{*}(z)=z^{2} \overline{r\left(\frac{1}{\bar{z}}\right)}
$$

Hence, if $A$ and $B$ are the zeros of $r(z)$, then $1 / \bar{A}$ and $1 / \bar{B}$ are the zeros of $r^{*}(z)$ and we write

$$
\tilde{\omega}(z)=\frac{(z-A)(z-B)}{(1-\bar{A} z)(1-\bar{B} z)} .
$$

Now we prove that $|\tilde{\omega}(z)|<1$ or equivalently that $A$ and $B$ lie inside or on the unit circle $|z|=1$. For this we apply the Cohn's rule to the polynomial $r$. Since $|a b|<1$ we see that

$$
r_{1}(z)=\frac{r(z)-a b r^{*}(z)}{z}=(1-a b)\left[(1+a b) z+\frac{1}{2}(a b-3 a-3 b+1)\right]
$$

has a zero at

$$
z_{0}=\frac{3}{2}\left(\frac{a+b}{1+a b}\right)-\frac{1}{2} .
$$

The zero $z_{0}$ lies inside or on the unit circle $|z|=1$ if $(a+b) /(1+a b) \in[-1 / 3,1)$. This proves the result.

Taking $b=a$ in Theorem 3.2, we get following result: 
Corollary 3.3. Let $f_{j} \in S\left(H^{a}\right)$ be CHD with the dilatation $\omega_{f_{j}}(z)=(a-z) /(1-a z)$ for each $j=1,2$. Then for any $\mu_{j}\left(\mu_{j} \in \mathbb{C},\left|\mu_{j}\right|=1, j=1,2\right), f_{\mu_{1}} * f_{\mu_{2}} \in S_{\mathcal{H}}$ is convex in the direction of $\overline{\mu_{1} \mu_{2}}$ for $a \in[-3+2 \sqrt{2}, 1)$.

The next result gives a condition for the directional convexity of the convolution $f_{\mu_{1}} * F_{\mu_{2}}$ when $f=h+\bar{g} \in S\left(H^{0}\right)$ with the dilatation $\omega_{f}=-z$ and $F=H+\bar{G} \in S\left(H^{b}\right)$ with the dilatation $\omega_{F}=\left(b-z e^{i \phi}\right) /\left(1-b z e^{i \phi}\right)(-1<b<1, \phi \in \mathbb{R})$.

Theorem 3.4. Let the function $f=h+\bar{g} \in S\left(H^{0}\right)$ and the function

$$
F=H+\bar{G} \in S\left(H^{b}\right)
$$

be CHD maps with the dilatations

$$
\omega_{f}(z)=-z \text { and } \omega_{F}(z)=\left(b-z e^{i \phi}\right) /\left(1-b z e^{i \phi}\right)(-1<b<1, \phi \in \mathbb{R}),
$$

respectively. Then for any $\mu_{j}\left(\mu_{j} \in \mathbb{C},\left|\mu_{j}\right|=1, j=1,2\right), f_{\mu_{1}} * F_{\mu_{2}} \in S_{\mathcal{H}}$ is convex in the direction of $\overline{\mu_{1} \mu_{2}}$ if any one of the following case holds:

(i) $\cos \phi=1$ and $-1 / 3 \leq b<1$

(ii) $-1 \leq \cos \phi<1$ and $\bar{b}^{2}<1 /(5+4 \cos \theta)$.

Proof. From (2.19), it follows that the dilatation $\tilde{\omega}$ of $f * F$ is given by

$$
\tilde{\omega}(z)=\frac{-2 z \omega_{F}\left(1+\omega_{F}\right)-z(1-z) \omega_{F}^{\prime}}{2\left(1+\omega_{F}\right)-z(1-z) \omega_{F}^{\prime}},
$$

where $\omega_{F}(z)=\left(b-z e^{i \phi}\right) /\left(1-b z e^{i \phi}\right)$ and $\omega_{F}^{\prime}(z)=\left(b^{2}-1\right) e^{i \phi} /\left(1-b e^{i \phi} z\right)^{2}(-1<b<1$, $\phi \in \mathbb{R})$. Hence, we have

$$
\tilde{\omega}(z)=-z e^{2 i \phi} \frac{t(z)}{t^{*}(z)}
$$

where

$$
t(z)=z^{2}-\frac{1+3 b}{2} e^{-i \phi} z+\frac{\left[2 b-(1-b) e^{i \phi}\right] e^{-2 i \phi}}{2}
$$

and

$$
t^{*}(z)=1-\frac{1+3 b}{2} e^{i \phi} z+\frac{\left[2 b-(1-b) e^{-i \phi}\right] e^{2 i \phi}}{2} .
$$

We need only to show that $|\tilde{\omega}(z)|<1$. (i) If $\cos \phi=1$, then

$$
t(z)=z^{2}-\frac{1+3 b}{2} z+\frac{3 b-1}{2}=(z-1)\left(z-\frac{3 b-1}{2}\right)
$$

and

$$
t^{*}(z)=(1-z)\left(1-\frac{3 b-1}{2} z\right) .
$$

Hence, the dilatation $\tilde{\omega}$ of $f * F$ becomes

$$
\tilde{\omega}(z)=-z \frac{(z-1)}{(1-z)} \frac{\left(z-\frac{3 b-1}{2}\right)}{\left(1-\frac{3 b-1}{2} z\right)}
$$


which imply that $|\tilde{\omega}(z)|<1$ when $|(3 b-1) / 2| \leq 1$ or when $-1 / 3 \leq b<1$. This proves the result if case (i) holds.

(ii)If $-1 \leq \cos \phi<1$, choose

$$
a_{0}=\frac{\left[2 b-(1-b) e^{i \phi}\right] e^{-2 i \phi}}{2} \quad \text { and } \quad a_{1}=-\frac{(1+3 b) e^{-i \phi}}{2} .
$$

Then $t(z)=z^{2}+a_{1} z+a_{0}$ and in this case, we have

$$
\begin{aligned}
1-\left|a_{0}\right|^{2} & =1-\frac{\left[2 b-(1-b) e^{i \phi}\right]\left[2 b-(1-b) e^{-i \phi}\right]}{4} \\
& =\frac{1-b}{4}[b(5+4 \cos \phi)+3]>0
\end{aligned}
$$

when $b>-3 /(5+4 \cos \phi)$. Under the condition when $b>-3 /(5+4 \cos \phi)$, we apply Cohn's rule to the polynomial $t(z)$. Consider

$$
t_{1}(z)=\frac{t(z)-a_{0} t^{*}(z)}{z}=\frac{1-b}{4}[b(5+4 \cos \phi)+3]\left(z-z_{0}\right),
$$

where

We have

$$
z_{0}=\frac{(1+3 b)\left(1+2 e^{-i \phi}\right)}{3+b(5+4 \cos \phi)}=: \frac{u(b)}{v(b)} .
$$

$$
|v(b)|^{2}-|u(b)|^{2}=4(1-\cos \phi)\left[1-b^{2}(5+4 \cos \phi)\right]>0
$$

when

Since

$$
b^{2}<\frac{1}{5+4 \cos \phi} \quad \text { or } \quad-\frac{1}{\sqrt{5+4 \cos \phi}}<b<\frac{1}{\sqrt{5+4 \cos \phi}} \text {. }
$$

$$
b^{2}<\frac{1}{5+4 \cos \phi} \Rightarrow b>-\frac{3}{5+4 \cos \phi}
$$

when $-1 \leq \cos \phi<1$, the result is proved if case (ii) holds.

For $b=0$, Theorem 3.4, reduces to the following simpler form:

Corollary 3.5. Let $f, F \in S\left(H^{0}\right)$ with the dilatations

$$
\omega_{f}(z)=-z \text { and } \omega_{F}(z)=-z e^{i \phi}(\phi \in \mathbb{R}),
$$

respectively. Then for any $\mu_{j}\left(\mu_{j} \in \mathbb{C},\left|\mu_{j}\right|=1, j=1,2\right), f_{\mu_{1}} * F_{\mu_{2}} \in S_{\mathcal{H}}$ is convex in the direction of $\overline{\mu_{1} \mu_{2}}$.

Further, for $\phi=\pi+\theta$ Theorem 3.4 takes the following form:

Corollary 3.6. Let the function $f=h+\bar{g} \in S\left(H^{0}\right)$ with the dilation $\omega_{f}(z)=-z$ and let the function $F=H+\bar{G} \in S\left(H^{b}\right)$ with the dilatation

$$
\omega_{F}(z)=\left(b+z e^{i \theta}\right) /\left(1+b z e^{i \theta}\right)(-1<b<1, \theta \in \mathbb{R}) .
$$

Then for any $\mu_{j}\left(\mu_{j} \in \mathbb{C},\left|\mu_{j}\right|=1, j=1,2\right), f_{\mu_{1}} * F_{\mu_{2}} \in S_{\mathcal{H}}$ is convex in the direction of $\overline{\mu_{1} \mu_{2}}$ if any one of the following case holds:

(i) $\cos \theta=-1$ and $-1 / 3 \leq b<1$

(ii) $-1<\cos \theta \leq 1$ and $b^{2}<1 /(5-4 \cos \theta)$. 


\section{Examples}

In this section, we give following examples to illustrate our main results. Examples 1 and 2 are based on Theorem 3.1, Example 3 is on Theorem 3.2 and Example 4 is on Theorem 3.4 .

Example 4.1. Let for some $\mu_{1}, \mu_{2} \in \mathbb{C}$ and for some $a(-1<a<1)$,

$$
f_{\mu_{1}}(z)=h(z)+\overline{g(z)} \in S\left(H^{a}\right),
$$

where

$$
h(z)=\frac{2 z-(1+a) \mu_{1} z^{2}}{2\left(1-\mu_{1} z\right)^{2}} \text { and } g(z)=\frac{2 a z-(1+a) \mu_{1} z^{2}}{2\left(1-\mu_{1} z\right)^{2}}
$$

and $F_{\mu_{2}}(z)=H(z)+\overline{G(z)} \in S\left(H^{0}\right)$, where

$$
H(z)=\frac{2 z-\mu_{2} z^{2}}{2\left(1-\mu_{2} z\right)^{2}} \text { and } G(z)=\frac{-\mu_{2} z^{2}}{2\left(1-\mu_{2} z\right)^{2}} .
$$

Then $f_{\mu_{1}} * F_{\mu_{2}} \in S_{\mathcal{H}}$ if $a \in[-1 / 3,1)$ and is convex in the direction of $\overline{\mu_{1} \mu_{2}}$.

Example 4.2. Let for some $\mu_{1}, \mu_{2} \in \mathbb{C}$ and for some $a(-1<a<1)$,

$$
f_{\mu_{1}}(z)=h(z)+\overline{g(z)} \in S\left(H^{a}\right),
$$

where

$$
h(z)=\frac{2 z-(1+a) \mu_{1} z^{2}}{2\left(1-\mu_{1} z\right)^{2}} \text { and } g(z)=\frac{2 a z-(1+a) \mu_{1} z^{2}}{2\left(1-\mu_{1} z\right)^{2}}
$$

and $F_{\mu_{2}}(z)=H(z)+\overline{G(z)} \in S\left(H^{0}\right)$, where

$H(z)=\frac{\overline{\mu_{2}}}{8} \log \frac{1+\mu_{2} z}{1-\mu_{2} z}+\frac{3 z-2 \mu_{2} z^{2}}{4\left(1-\mu_{2} z\right)^{2}}$ and $G(z)=-\frac{\overline{\mu_{2}}}{8} \log \frac{1+\mu_{2} z}{1-\mu_{2} z}+\frac{z-2 \mu_{2} z^{2}}{4\left(1-\mu_{2} z\right)^{2}}$.

Then $f_{\mu_{1}} * F_{\mu_{2}} \in S_{\mathcal{H}}$ if $a \in[0,1)$ and is convex in the direction of $\overline{\mu_{1} \mu_{2}}$.

Example 4.3. Let for some $\mu_{1}, \mu_{2} \in \mathbb{C}$ and for some $a(-1<a<1)$,

$$
f_{\mu_{1}}(z)=h(z)+\overline{g(z)} \in S\left(H^{a}\right),
$$

where

$$
h(z)=\frac{2 z-(1+a) \mu_{1} z^{2}}{2\left(1-\mu_{1} z\right)^{2}} \text { and } g(z)=\frac{2 a z-(1+a) \mu_{1} z^{2}}{2\left(1-\mu_{1} z\right)^{2}}
$$

and $F_{\mu_{2}}(z)=H(z)+\overline{G(z)} \in S\left(H^{1 / 2}\right)$, where

$$
H(z)=\frac{4 z-3 \mu_{2} z^{2}}{4\left(1-\mu_{2} z\right)^{2}} \text { and } G(z)=\frac{2 z-3 \mu_{2} z^{2}}{4\left(1-\mu_{2} z\right)^{2}} .
$$

Then $f_{\mu_{1}} * F_{\mu_{2}} \in S_{\mathcal{H}}$ if $a \in[-5 / 7,1)$ and is convex in the direction of $\overline{\mu_{1} \mu_{2}}$.

Example 4.4. Let for some $\mu_{1}, \mu_{2} \in \mathbb{C}$ and for some $b(-1<b<1)$,

$$
f_{\mu_{1}}(z)=h(z)+\overline{g(z)} \in S\left(H^{0}\right)
$$

where

$$
h(z)=\frac{2 z-\mu_{1} z^{2}}{2\left(1-\mu_{1} z\right)^{2}} \quad \text { and } g(z)=\frac{-\mu_{1} z^{2}}{2\left(1-\mu_{1} z\right)^{2}} .
$$


and $F_{\mu_{2}}(z)=H(z)+\overline{G(z)} \in S\left(H^{b}\right)$, where

$$
H(z)=\frac{1-b}{4} \overline{\mu_{2}} \log \frac{1+\mu_{2} z}{1-\mu_{2} z}+\frac{1+b}{2} \frac{z}{1-\mu_{2} z}
$$

and

$$
G(z)=-\frac{1-b}{4} \overline{\mu_{2}} \log \frac{1+\mu_{2} z}{1-\mu_{2} z}+\frac{1+b}{2} \frac{z}{1-\mu_{2} z} .
$$

Then $f_{\mu_{1}} * F_{\mu_{2}} \in S_{\mathcal{H}}$ and is convex in the direction of $\overline{\mu_{1} \mu_{2}}$.

Acknowledgement. The authors are thankful to the referee for suggesting to add some examples based on the technique used in our Main Results.

\section{References}

[1] Beig, S., Ravichandran, V., Convolution and convex combination of harmonic mappings, Bull. Iranian Math. Soc., 45(2019), no. 5, 1467-1486.

[2] Clunie, J., Sheil-Small, T., Harmonic univalent functions, Ann. Acad. Sci. Fenn. Ser. A I Math., 9(1984), 3-25.

[3] Dorff, M., Convolutions of planar harmonic convex mappings, Complex Variables Theory Appl., 45(2001), no. 3, 263-271.

[4] Dorff, M., Nowak, M., Wołoszkiewicz, M., Convolutions of harmonic convex mappings, Complex Var. Elliptic Equ., 57(2012), no. 5, 489-503.

[5] Dorff, M., Rolf, J.S., Anamorphosis, mapping problems, and harmonic univalent functions, in: Explorations in Complex Analysis, 197-269, Classr. Res. Mater. Ser, Math. Assoc. America, Washington, DC.

[6] Kumar, R., et al., Convolution properties of some harmonic mappings in the right halfplane, Bull. Malays. Math. Sci. Soc., 39(2016), no. 1, 439-455.

[7] Kumar, R., Gupta, S., Singh, S., Convolution properties of a slanted right half-plane mapping, Mat. Vesnik, 65(2013), no. 2, 213-221.

[8] Lewy, H., On the non-vanishing of the Jacobian in certain one-to-one mappings, Bull. Amer. Math. Soc., 42(1936), no. 10, 689-692.

[9] Li, Y., Liu, Z., Convolutions of harmonic right half-plane mappings, Open Math., 14(2016), no. 1, 789-800.

[10] Li, L., Ponnusamy, S., Convolutions of slanted half-plane harmonic mappings, Analysis (Munich), 33(2013), no. 2, 159-176.

[11] Li, L., Ponnusamy, S., Solution to an open problem on convolutions of harmonic mappings, Complex Var. Elliptic Equ., 58(2013), no. 12, 1647-1653.

[12] Li, L., Ponnusamy, S., Note on the convolution of harmonic mappings, Bull. Aust. Math. Soc., 99(2019), no. 3, 421-431.

[13] Liu, Z., Ponnusamy, S., Univalency of convolutions of univalent harmonic right halfplane mappings, Comput. Methods Funct. Theory, 17(2017), no. 2, 289-302.

[14] Pommerenke, C., On starlike and close-to-convex functions, Proc. London Math. Soc., 13(1963), no. 3, 290-304.

[15] Rahman, Q.I., Schmeisser, G., Analytic Theory of Polynomials, London Mathematical Society Monographs. New Series, 26, The Clarendon Press, Oxford University Press, Oxford, 2002. 
Poonam Sharma

Department of Mathematics \& Astronomy, University of Lucknow,

Lucknow 226007 India

e-mail: sharma_poonam@lkouniv.ac.in

Omendra Mishra

Department of Mathematics \& Astronomy, University of Lucknow,

Lucknow 226007 India

e-mail: mishraomendra@gmail.com 